\title{
Loop Entanglement of Semicrystalline Polyethylene in Amorphous Region: Diamond Lattice Approach
}

\author{
ZHONG-HUI DUAN, ${ }^{1}$ LOUIS N. HOWARD ${ }^{2}$ \\ ${ }^{1}$ Department of Mathematics, University of Michigan, Ann Arbor, Michigan 48109 \\ ${ }^{2}$ Department of Mathematics, Florida State University, Tallahassee, Florida 32306
}

Received 22 June 1998; accepted 6 October 1998

\begin{abstract}
Linear polyethylenes in the amorphous region have been simulated as restricted random walks on a diamond lattice between two absorbing planes. The links among loops were studied by treating loops as oriented curves. The local conformations of polyethylene chains (i.e., trans and gauche energy differences) were considered in the simulation, thereby determining the effect of crystallization temperature on the loop entanglement. It was found that the total Gauss winding and link density of linked loops increased with the thickness of the amorphous region. This result agrees with that of the cubic lattice model. The link probability decreases very slowly with the thickness of the amorphous region. On the other hand, the results presented clearly indicate that all statistical measures of linked loops decrease with temperature. (c) 1999 John Wiley \& Sons, Inc. J Comput Chem 20: 348-353, 1999
\end{abstract}

Keywords: random walks; diamond lattice; semicrystalline polyethylene; loop entanglement; computer simulation

\section{Introduction}

The tensile properties of semicrystalline polyethylene have been studied extensively by Mandelkern et al. ${ }^{1-5}$ It has been found that the stress-strain curves depend greatly on the molecular weight, crystallinity, and thickness of the amorphous region-and therefore on the entanglement of polyethylene chains in the amorphous region. ${ }^{1-6}$ To get a better understanding of the "entangle-

Correspondence to: Z.-H. Duan ment," Guttman et al. ${ }^{7}$ proposed to regard a chain in the amorphous region as a random walk on a cubic lattice between two parallel absorbing planes representing adjacent crystallites. Using solutions to the classical "gambler's ruin" problem, they were able to compute the statistics of the random walks, which are, presumably, statistics of polyethylene chains in the amorphous region. Lacher et al. used this cubic lattice model, together with computer simulations, to study some of the statistics of connections by linked loops. They introduced three statistical measures: link probability 
$\left(P_{\text {link }}\right)$, the probability that a loop links some loop from the opposing plane; link density $\left(D_{\text {link }}\right)$, the expected number of opposing loops that a given loop links; and total Gauss winding $(W)$, the sum of the absolute values of the linking numbers that a loop forms with all the loops from the opposing plane. They found that these measures remain constant or increase with the thickness of the amorphous region. In addition to the simulation results, they computed $P_{\text {link }}$ and $D_{\text {link }}$ analytically using a simplified model. The asymptotic estimates of the simplified model and their simulation results agree within $10 \%$. These results provided a new insight in understanding the connections between crystalline lamellae and thus the molecular mechanisms underlying the deformation of semicrystalline polyethylene..$^{8-10}$

The polyethylene chain does not actually fit on a cubic lattice. To make some physical sense of each step of the random walks, and to include the temperature effects on the chain statistics, in our previous work, we extended the original cubic lattice model and modeled the chains as restricted random walks on a diamond lattice. ${ }^{11}$ We present here the estimations of the three statistical measures of linked loops $-P_{\text {link }}, D_{\text {link }}$, and $W$, using restricted random walks on a diamond lattice with two absorbing boundary planes.

\section{Diamond Lattice and Random Walks}

The model of an amorphous region is adapted from our previous work. ${ }^{11}$ The polyethylene chains are modeled as restricted random walks on a diamond lattice between two absorbing planes. The lattice angle of $109.47^{\circ}$ resembles the tetrahedral angle between two adjacent $\mathrm{C}-\mathrm{C}$ bonds. The lattice bonds (resemble $\mathrm{C}-\mathrm{C}$ bonds) form a vector set, $\mathbf{B}=\left\{\mathbf{b}_{1}, \mathbf{b}_{2}, \mathbf{b}_{3}, \mathbf{b}_{4}, \mathbf{b}_{5}, \mathbf{b}_{6}, \mathbf{b}_{7}, \mathbf{b}_{8}\right\}$, where (see Fig. 1):

$$
\begin{aligned}
& \mathbf{b}_{1}=(0, \sqrt{1 / 3}, \sqrt{2 / 3}), \\
& \mathbf{b}_{2}=(0, \sqrt{1 / 3},-\sqrt{2 / 3}), \\
& \mathbf{b}_{3}=(0,-\sqrt{1 / 3}, \sqrt{2 / 3}) \\
& \mathbf{b}_{4}=(0,-\sqrt{1 / 3},-\sqrt{2 / 3}), \\
& \mathbf{b}_{5}=(\sqrt{2 / 3}, \sqrt{1 / 3}, 0), \\
& \mathbf{b}_{6}=(\sqrt{2 / 3},-\sqrt{1 / 3}, 0) \\
& \mathbf{b}_{7}=(-\sqrt{2 / 3}, \sqrt{1 / 3}, 0), \\
& \mathbf{b}_{8}=(-\sqrt{2 / 3},-\sqrt{1 / 3}, 0)
\end{aligned}
$$

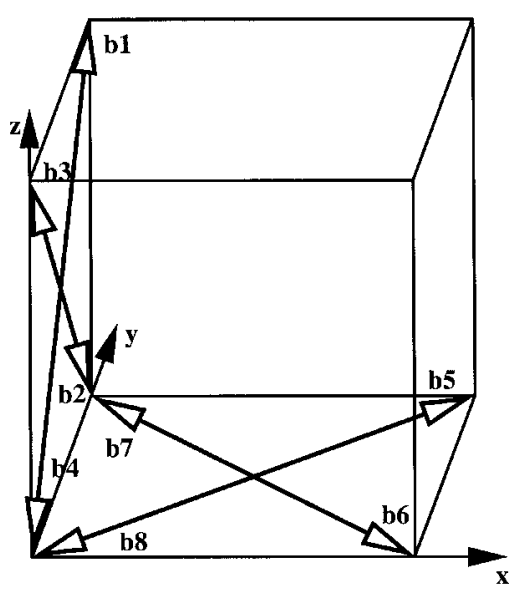

FIGURE 1. The vector representations of the eight lattice bonds of a diamond lattice.

For computational convenience, it can be rescaled to be $\mathbf{A}=\left\{\mathbf{a}_{1}, \mathbf{a}_{2}, \mathbf{a}_{3}, \mathbf{a}_{4}, \mathbf{a}_{5}, \mathbf{a}_{6}, \mathbf{a}_{7}, \mathbf{a}_{8}\right\}$, where $\mathbf{a}_{1}=(0,1,1), \mathbf{a}_{2}=(0,1,-1), \mathbf{a}_{3}=(0,-1,1), \mathbf{a}_{4}=$ $(0,-1,-1), \quad \mathbf{a}_{5}=(1,1,0), \mathbf{a}_{6}=(1,-1,0), \quad \mathbf{a}_{7}=$ $(-1,1,0), \mathbf{a}_{8}=(-1,-1,0)$. The two parallel absorbing boundary planes at $z=0$ and $z=M$ resemble the two adjacent crystalline lamellae, separated by $\mathrm{M} \mathrm{C}-\mathrm{C}$ bonds. Consider a random walk on a diamond lattice initiated at a site $(x, y, 0)$ on $z=0$. The first step of the walk takes either $\mathbf{a}_{1}$ or $\mathbf{a}_{3}$ with equal probabilities to step into the amorphous region and forms a trans with its two preceding bonds in the crystal. (i.e., the interface is assumed to be normal to the crystalline chains in the crystal). The walk is continued on the lattice with a certain probability of preceding to one of the three adjacent lattice points to form a sequence of rotational isomers (i.e., trans, gauche ${ }^{+}$or gauche $\left.^{-}\right)$. The probabilities for every three steps to form a trans, gauche ${ }^{+}$, or gauche ${ }^{-}$have been determined to be:

$$
\begin{gathered}
p(t)=(1+2 \sigma) /\left(1+4 \sigma+2 \sigma^{2}+2 \sigma^{2} \omega\right), \\
p\left(g^{+}\right)=(1-p(t)) / 2, \quad p\left(g^{-}\right)=(1-p(t)) / 2,
\end{gathered}
$$

respectively, where $\sigma=e^{-E \sigma / R T}, E_{\sigma}=430-590 \mathrm{cal}$ $\mathrm{mol}^{-1}, \omega=e^{-E \omega / R T}$, and $E_{\omega}=1700-2000 \mathrm{cal}$ $\mathrm{mol}^{-1}$, and $T$ stands for the temperature. ${ }^{11}$ The walk terminates when it reaches the plane from which it starts (where a loop is formed) or the opposite plane (where a tie is formed) (Fig. 2). For the walk initiated at $(x, y, M)$, the first step will take $\mathbf{a}_{2}$ or $\mathbf{a}_{4}$, instead of $\mathbf{a}_{1}$ or $\mathbf{a}_{3}$, to step into the amorphous region. The thickness of the amorphous region is between $50 \AA$ and $200 \AA^{12}$ and the length of the $\mathrm{C}-\mathrm{C}$ bonds is about $1.54 \AA$; 


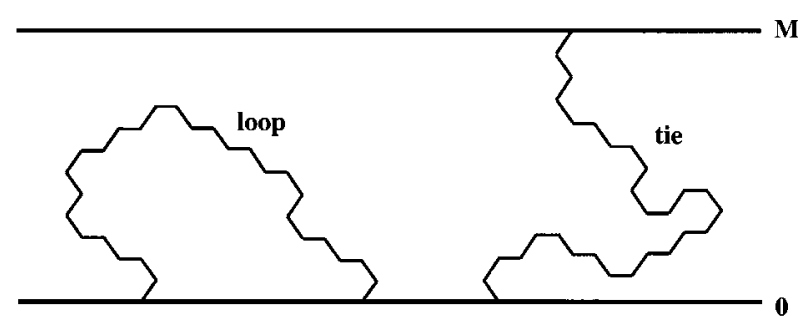

FIGURE 2. Projections of two random walks on a diamond lattice on the $Y Z$ plane (a loop re-enters the same crystal as it emerges, and a tie connects the two adjacent crystals directly).

therefore, reasonable values for $M$ are between 40 and 160.

\section{Linking and Linking Number}

In this study, we use some notations similar to those in ref. 10. A walk started at site $(x, y, 0)$ is called an L-walk (lower walk). A walk started at site $(x, y, M)$ is called a $U$-walk (upper walk). The L-walk that is a loop is called an L-loop. The U-walk that is a loop is called a U-loop (Fig. 3). A U-loop and an L-loop may link with each other. The linking number between them was studied by treating them as two oriented closed curves, $\mathrm{L}$ and $\mathrm{U}$. A segment from $\mathrm{L}$ and one from $\mathrm{U}$ determine an undercrossing if the projection of the segment from $\mathrm{L}$ crosses under the projection of the segment from $\mathrm{U}$. The value of the undercrossing is determined by the righthand rule shown in Figure $4 .{ }^{8-10}$ The sum of the values of all undercrossings of $\mathrm{L}$ with $U$ is defined to be the linking number of the two oriented closed curves. If it is not zero, we say $\mathrm{L}$ and $\mathrm{U}$ are linked. The linking number of two intersected curves is undefined.

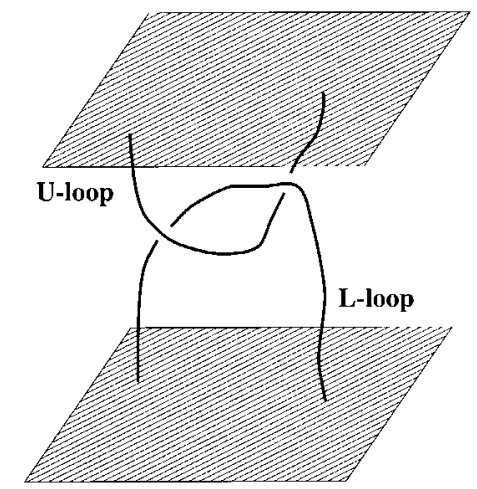

FIGURE 3. An L-loop is linked with a U-loop.

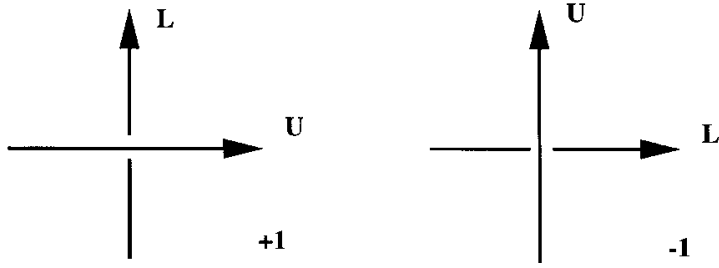

FIGURE 4. The undercrossing of two segments from $L$ and $U$.

\section{Computer Simulation}

The simulation starts with generating a set of U-walks initiated from the starting set $\left\{\left(x_{i}, x_{j}\right.\right.$, $M)\}$ with certain starting probability (detailed later). The paths of the U-loops in the set of U-walks are recorded. Then, a sequence of L-walks from $(0,0,0)$ is generated. For each L-loop in the sequence, the linking number of the L-loop with each of the U-loops is calculated and the three statistical measures, $P_{\text {link }}, D_{\text {link }}$, and $W$, are updated. The process is repeated until the statistical estimations of $P_{\text {link }}, D_{\text {link }}$, and $W$ are stabilized. Because the polyethylene chains are simulated as restricted random walks on a diamond lattice defined by vector set $\mathbf{A}$, the starting set must be a set of triplets of integers. However, it should not be a set of all triplets of integers on $z=M$ plane, because: (1) The probability is quite small for an L-loop starting from $(0,0,0)$ to link with a U-loop, which starts far away from $(0,0, M)$. Lacher et al. has confirmed that most linking $(90 \%)$ for a loop on a cubic lattice occurs within $M$ lateral units of the beginning point of the loop. ${ }^{8-10}$ We examined the size influence on linking for small values of $M$ and found that most linking for a loop on a diamond lattice occurs within $4 M$ lateral units of the beginning point of the loop. (2) The polyethylene crystal structure consists of repeated cell units (see Fig. 5) with dimensions of $4.946 \AA, 7.418 \AA$, and $2.546 \AA$ in $x, y$, and $z$ directions, respectively ${ }^{13}$ and the polyethylene molecules lie parallel to the $z$ axis in the crystal. Hence, we consider the starting positions of all U-loops being restricted within a big square with dimensions $10 M \times 10 M$ on the $z=M$ plane centered at $(0,0, M)$. To best fit the polyethylene chains in the crystal, the cell unit in the $x$-direction in the simulation is taken to be:

$$
\frac{4.946}{1.54 \sqrt{2 / 3}}(\approx 4)
$$




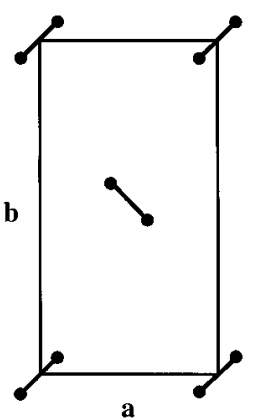

FIGURE 5. A schematic view of the projection of a unit cell to the crystal face ( $\bullet$ carbon atoms; $-\mathrm{C}-\mathrm{C}$ bonds; $a \approx 4.946 \AA ; \quad b \approx 7.418 \AA$ ).

steps and in the $y$-direction it is taken to be:

$$
\frac{7.418}{1.54 \sqrt{1 / 3}}(\approx 8)
$$

steps (because all the steps of a random walk are actually from the vector set $\mathbf{B}$ and each step resembles one $\mathrm{C}-\mathrm{C}$ bond, which has a length of $1.54 \AA$ ). Therefore, the starting set of the U-walks is chosen to be $\left\{\left(x_{i}, y_{j}, M\right)\right\}$, where $x_{i}$ is an even number between $-5 M$ and $5 M$ and $y_{j}$ is taken to be a multiple of eight between $-5 M$ and $5 M$ if $x_{i}$ is a multiple of four, otherwise, to be a multiple of eight plus four between $-5 \mathrm{M}$ and $5 \mathrm{M}$.

The starting probability is taken to meet the density requirement such that the density in the crystal is $1 \mathrm{~g} / \mathrm{cm}^{3}$ and the density in the amorphous region is about $0.853 \mathrm{~g} / \mathrm{cm}^{3}$. ${ }^{14}$ Table I shows the starting probabilities used at different temperatures. They are obtained by dividing $0.853 \mathrm{M}$ by two times the expected length of a polyethylene chain in the amorphous region. ${ }^{11}$

Clearly, an L-loop and a U-loop can be treated as two piecewise linear-oriented closed curves. The projection of a loop into the $Y Z$ plane is a piecewise linear path linked through the following vectors $(1,0),(-1,0),(1,1),(-1,1),(1,-1)$, and

TABLE I. Starting Probabilities at Different Temperatures

\begin{tabular}{cr} 
Temperature $(\mathrm{K})$ & Starting Proba \\
\hline 300 & 0.18528 \\
350 & 0.18716 \\
400 & 0.18875 \\
450 & 0.19016 \\
500 & 0.19135 \\
$\infty$ & 0.21325
\end{tabular}

$(-1,-1)$. However, the paths of the L-loop and the U-loop just described might intersect with each other. To overcome the difficulty in computing the linking number, the path of an L-loop is offset by subtracting $1 / 4$ from both the $y$ coordinates and the $z$ coordinates of the path. The offset linking number of an L-loop and a U-loop can be calculated by applying the signed undercrossing method to the U-loop and the offset L-loop.

In what follows we examine some of the more technical details. It is very natural to think that we can compute the three measures by generating the U-walks and saving the paths of U-loops, then generating an L-loop and calculating the linking numbers by projecting every step of the L-loop and every step of each of the U-loops. However, the implementation of this direct method is computationally very expensive. For a single L-loop, computing the linking numbers involves calculation of the undercrossing between each step of the L-loop (the average loop length is about $2 M$ ) and each step of U-loops (the number of U-loops is about $M^{2}$ ). Thus, it requires a prohibitive amount of CPU time for large values of $M$. To overcome the computational difficulty, we use an array of linked lists. The size of the array is $M \times 10 M$ (the size of the projection region on $Y Z$ plane), and the structure of the element in the array is defined to be:

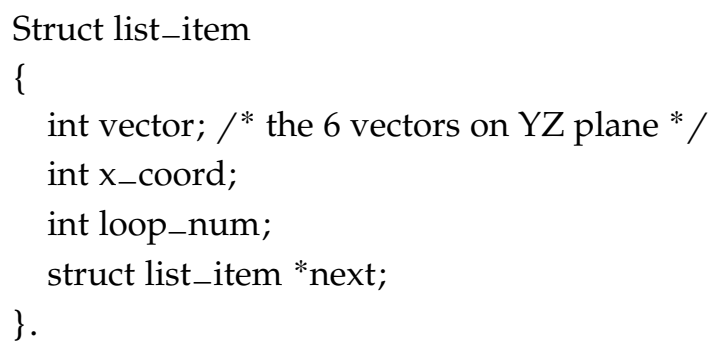

The projections of U-loops are saved in the linked lists. The linking number is computed using this array and the paths of the L-loops. The three statistics are updated at each step of an L-loop. The computer source code in $C$ is available from the authors upon request.

\section{Results and Discussion}

The results of simulation of 2000 runs are summarized in Figures 6-9. Each run consists of a set of U-loops and 100,000 L-loops. We can clearly see the dependence of the loop entanglement of polyethylene chains on both the thickness of the 
amorphous region and the crystallization temperature. The influence of the thickness of the amorphous region, $M$, on the statistics $P_{\text {link }}, D_{\text {link }}$, and $W$ at $400 \mathrm{~K}$ is shown in Figure 6. $P_{\text {link }}, D_{\text {link }}$, and $W$ increase with $M$. $P_{\text {link }}$ decreases with $M$ for $M>40$, but the rate of decrease is small as compared with the rate of decreasing fraction of ties $\left(\approx 4.4611 / M-M / 14.0158 / M^{2}\right)$. This result agrees with those obtained by Lacher et al. using cubic lattice model. ${ }^{8-10}$ It again emphasizes the role of linked loops in connecting two adjacent crystallites. The theoretical work on the entanglement complexity of a self-avoiding random polygon on a cubic lattice has shown that the absolute value of the writhe (an average of linking numbers ${ }^{15}$ ) of an $n$-edge polygon is almost always of order $o(\sqrt{n}) .^{16}$ It appears that our results agree with the theoretical conclusion in a qualitative sense [the average length of loops is $O(M)$; see ref. 11] and the

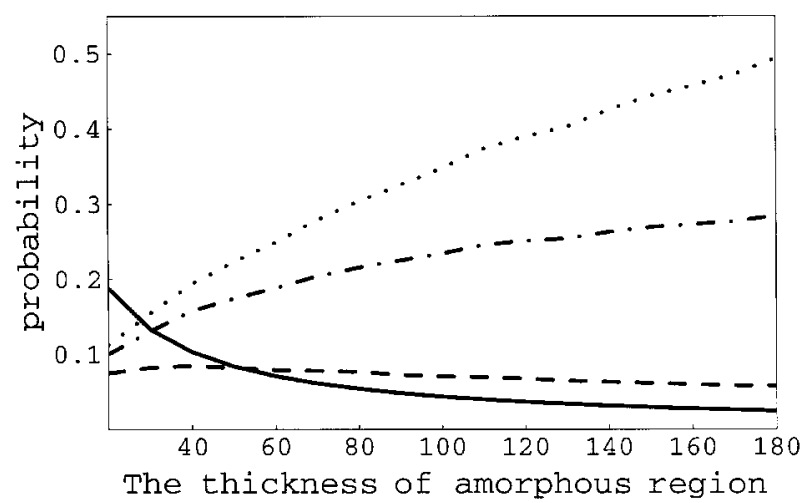

FIGURE 6. The effect of thickness of amorphous region on the statistics of linked loops (temperature taken to be $400 \mathrm{~K} ; \cdots W ;-\cdot-D_{\text {link }} ;---P_{\text {link }} ;-$ fraction of ties.

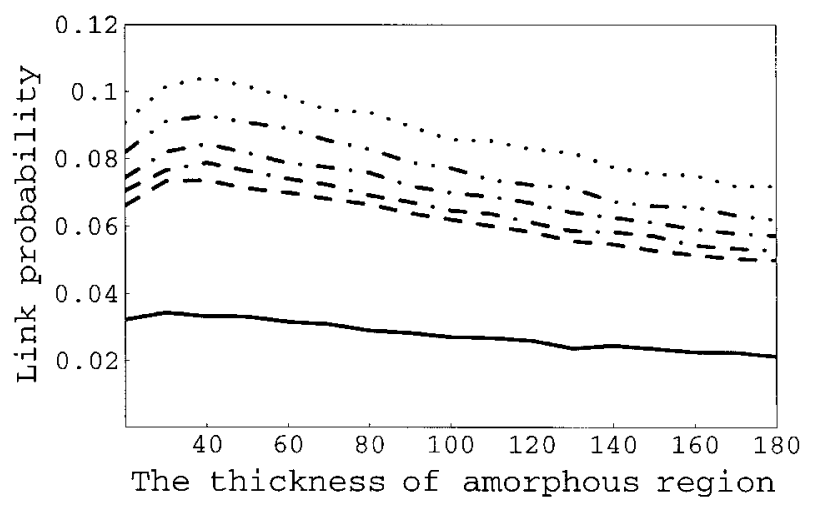

FIGURE 7. Temperature effect on link probability $(\cdots$ $T=300 \mathrm{~K} ;-\cdot \cdot-T=350 \mathrm{~K} ;-\cdot-T=400 \mathrm{~K} ;--\cdot T=450 \mathrm{~K}$; $--T=500 \mathrm{~K} ;-$ limit case $T=\infty$ ).

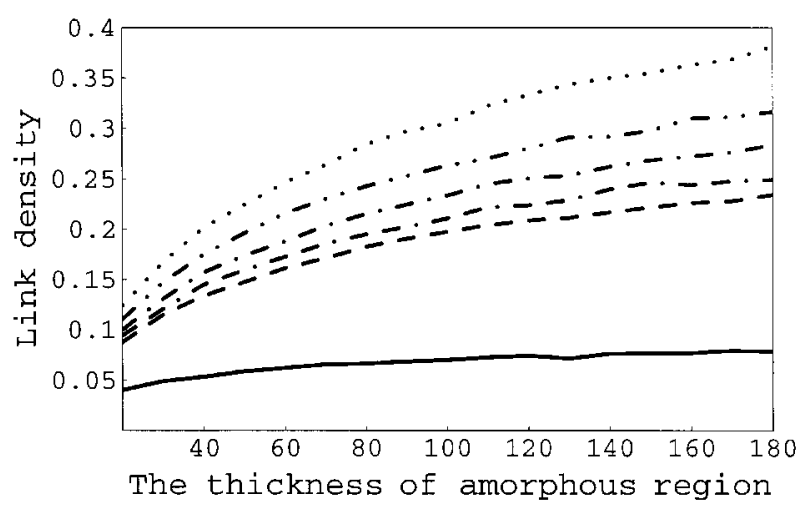

FIGURE 8. Temperature effect on link density $(\cdots T=$ $300 \mathrm{~K} ;-\cdot \cdot-T=350 \mathrm{~K} ;-\cdot-T=400 \mathrm{~K} ;--\cdot T=450 \mathrm{~K} ;--$ $T=500 \mathrm{~K} ;-$ limit case $T=\infty)$.

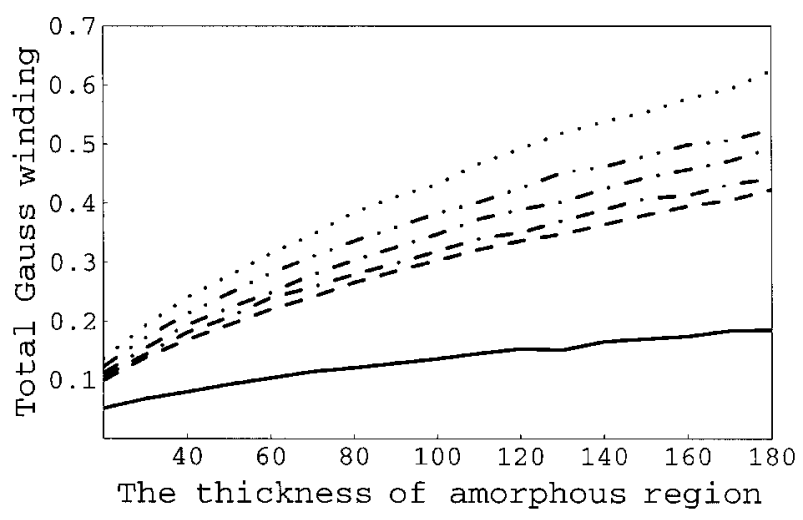

FIGURE 9. Temperature effect on total Gauss winding $(\cdots T=300 \mathrm{~K} ;-\cdot \cdot-T=350 \mathrm{~K} ;-\cdot-T=400 \mathrm{~K} ;--\cdot T=$ $450 \mathrm{~K} ;---T=500 \mathrm{~K} ;-$ limit case $T=\infty)$.

increasing properties of $D_{\text {link }}$ and $W$ with $M$ are independent of the lattice model.

The results of temperature dependence of the entanglement of polyethylene chains are shown in Figures 7-9. All the three statistics, $P_{\text {link }}, D_{\text {link }}$, and $W$, decrease as temperature increases. Theoretical calculation of the statistics of polyethylene chains indicates that more loops, with shorter average length, are formed at higher temperature and average length of ties is longer. ${ }^{11}$ The findings presented in Figures 7-9 are in good agreement with our intuition, based on theoretical resultsthat is, fewer loops are linked together at higher temperature.

It is known that the random walk models are not valid near the crystal surface. The polyethylene chain in the crystal region is conformationally ordered and the order persists over certain a number of steps along the chain (about 10 to $30 \AA$ ). ${ }^{12}$ The 
effects of the interphase on the statistics in the amorphous region will be examined in our future work.

\section{Acknowledgments}

Computations were performed at the Department of Mathematics, Florida State University. The authors thank Professors R. Alamo, R. C. Lacher, L. Mandelkern, and D. W. Sumners for helpful discussions and M. Boyd for helpful suggestions on compiling the source code.

\section{References}

1. Kennedy, M. A.; Peacock, A. J.; Mandelkern, L. Macromolecules 1994, 27, 5297.

2. Popli, R.; Mandelkern, L. J Polym Sci B Polym Phys 1987, $25,441$.

3. Mandelkern, L.; Smith, F. L.; Failla, M.; Kennedy, M. A.; Peacock, A. J. J Polym Sci B Polym Phys 1993, 31, 491.

4. Mandelkern, L.; Peacock, A. J. In: Lacher, R. C., ed. Studies in Physical and Theoretical Chemistry, Vol 54; Elsevier: Amsterdam, 1988, p 201.

5. Lucas, J. C.; Smith, F. L.; Mandelkern, L.; Peacock, A. J. Polym Engng Sci 1995, 35, 1117.

6. Alamo, R. G. Personal communication.

7. Guttman, C. M.; DiMarzio, E. A.; Hoffman, J. D. Polymer 1981, 22, 1466.

8. Lacher, R. C.; Bryant, J. L.; Howard, L. N. J Chem Phys 1986, 85, 6147.

9. Lacher, R. C.; Bryant, J. L.; Howard, L. N.; Sumners, L. W. Macromolecules 1986, 19, 2639.

10. Lacher, R. C. Macromolecules 1987, 20, 3054.

11. Duan, Z.-H.; Howard, L. N. J Chem Phys 1998, 108, 5608.

12. Mandelkern, L.; Alamo, R. G.; Kennedy, M. A. Macromolecules 1990, 23, 4721.

13. Young, R. J.; Lovell, P. A. Introduction of Polymers; Chapman \& Hall: London, 1991, p 251.

14. Chiang, R.; Flory, P. J. J Am Chem Soc 1961, 83, 2857.

15. Lacher, R. C.; Sumners, D. W. In: Roe, R. J., ed. Computer Simulation of Polymers; Prentice-Hall: Englewood Cliffs, NJ, 1991, p 365.

16. Janse van Rensburg, E. J.; Orlandin, E.; Sumners, D. W.; Tesi, M. C.; Whittington, S. G. In: Mesirov, J. P.; Schulten, K.; Sumners, D. W., eds. Mathematical Approaches to Biomolecular Structure and Dynamics; Springer: Berlin, 1996, p 21. 